\title{
Assembly of clathrates from tetrahedral patchy colloids with narrow patches
}

Cite as: J. Chem. Phys. 151, 094502 (2019); https://doi.org/10.1063/1.5109382

Submitted: 08 May 2019 . Accepted: 06 August 2019 . Published Online: 05 September 2019

Eva G. Noya (D), Itziar Zubieta, David J. Pine (D), and Francesco Sciortino (D)
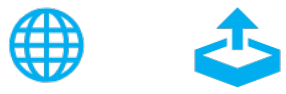

\section{ARTICLES YOU MAY BE INTERESTED IN}

Decoding signatures of structure, bulk thermodynamics, and solvation in three-body angle distributions of rigid water models

The Journal of Chemical Physics 151, 094501 (2019); https://doi.org/10.1063/1.5111545

Configurational entropy of glass-forming liquids

The Journal of Chemical Physics 150, 160902 (2019); https://doi.org/10.1063/1.5091961

Breakdown of the law of rectilinear diameter and related surprises in the liquid-vapor coexistence in systems of patchy particles

The Journal of Chemical Physics 150, 224510 (2019); https://doi.org/10.1063/1.5098551

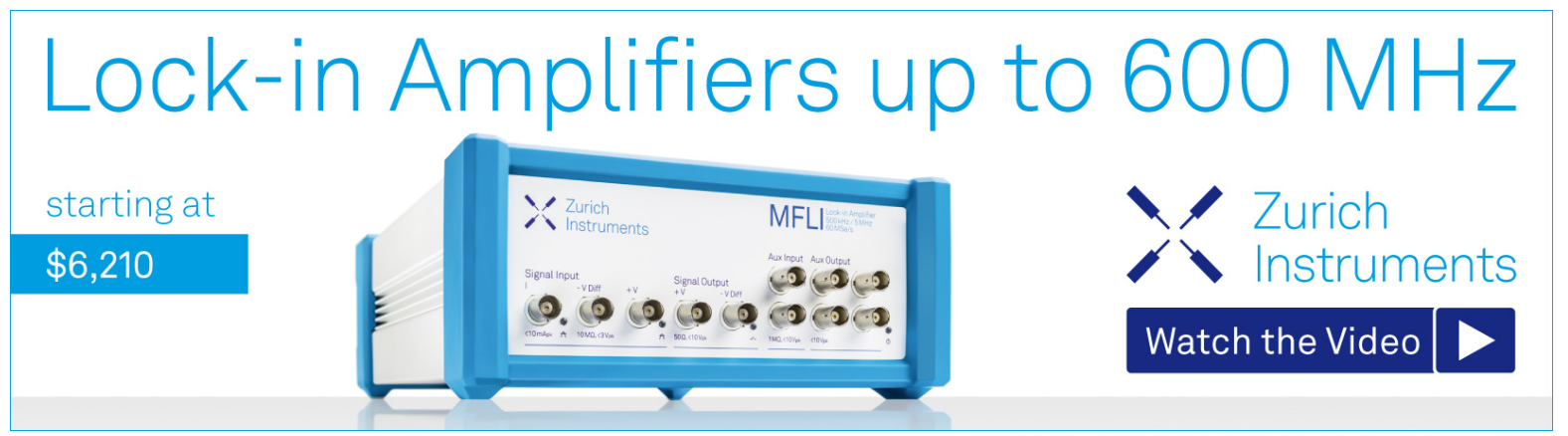

J. Chem. Phys. 151, 094502 (2019); https://doi.org/10.1063/1.5109382 


\title{
Assembly of clathrates from tetrahedral patchy colloids with narrow patches
}

\author{
Cite as: J. Chem. Phys. 151, 094502 (2019); doi: 10.1063/1.5109382 \\ Submitted: 8 May 2019 - Accepted: 6 August 2019 • \\ Published Online: 5 September 2019
}

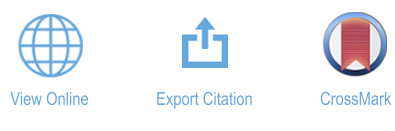

Eva G. Noya, ${ }^{1, a)}$ (D) Itziar Zubieta,' David J. Pine, ${ }^{2}$ (D) and Francesco Sciortino ${ }^{3, b)}$ (D)

\author{
AFFILIATIONS \\ ${ }^{1}$ Instituto de Química Física Rocasolano, Consejo Superior de Investigaciones Científicas, CSIC, Calle Serrano 119, \\ 28006 Madrid, Spain \\ ${ }^{2}$ Center for Soft Matter Research, Department of Physics, New York University, New York, New York 10003, USA \\ ${ }^{3}$ Department of Physics and CNR-ISC, Sapienza Universitá di Roma, Piazzale Aldo Moro 2, I-00185 Roma, Italy \\ a) Electronic mail: eva.noya@iqfr.csic.es \\ b) Electronic mail: francesco.sciortino@uniroma7.it
}

\begin{abstract}
Here, we revisit the assembly of colloidal tetrahedral patchy particles. Previous studies have shown that the crystallization of diamond from the fluid phase depends more critically on patch width than on the interaction range: particles with patches narrower than $40^{\circ}$ crystallize readily and those with wide patches form disordered glass states. We find that the crystalline structure formed from the fluid also depends on the patch width. Whereas particles with intermediate patches assemble into diamond (random stacking of cubic and hexagonal diamond layers), particles with narrow patches (with width $\approx 20^{\circ}$ or less) crystallize frequently into clathrates. Free energy calculations show that clathrates are never (in the pressure-temperature plane) thermodynamically more stable than diamond. The assembly of clathrate structures is thus attributed to kinetic factors that originate from the thermodynamic stabilization of pentagonal rings with respect to hexagonal ones as patches become more directional. These pentagonal rings present in the fluid phase assemble into sII clathrate or into large clusters containing 100 particles and exhibiting icosahedral symmetry. These clusters then grow by interpenetration. Still, the organization of these clusters into extended ordered structures was never observed in the simulations.
\end{abstract}

Published under license by AIP Publishing. https://doi.org/10.1063/1.5109382

\section{INTRODUCTION}

The assembly of patchy particles has been the subject of considerable research during the last years due to their potential usefulness for producing new materials. ${ }^{1-3}$ Among the plethora of geometries, tetrahedral patchy particles have been probably the system attracting more attention. ${ }^{4-16}$ Some of these studies were motivated by the desire to obtain a colloidal diamond crystal (a material with interesting photonic properties $)^{4-10,16}$ but also by the potential usefulness of simple colloidal models to understand the behavior of more complex systems, in particular, that of tetrahedral atomic and molecular liquids, such as water, carbon, silicon, germanium, etc. $^{13-15,17}$

After these extensive studies, the phase diagram and the assembly behavior of tetrahedral patchy particles are relatively well understood. Evaluation of the equilibrium phase diagram showed that diamond competes with a body centered cubic (BCC) solid, in which the particles are bound to four of the eight nearest neighbors forming two interpenetrated diamond lattices. ${ }^{6-8,11}$ These studies also found that the thermodynamic stability of diamond over that of the BCC becomes larger for shorted ranged models. ${ }^{6-8}$ In spite of being the thermodynamically stable phase at low pressure and temperature, simulations have shown that diamond is not always readily accessible from the fluid phase as the system can fall into kinetic traps in which a glass state is formed instead. A systematic study of the parameter space found that the interaction range is the relevant parameter to make diamond kinetically accessible. Particles with patch widths narrower than $30^{\circ}$ assemble into a crystal, consisting in a random stack of cubic (that contains only chair 6-membered rings) and hexagonal (with both chair and boat rings) diamond layers, and into a disordered glass structure for particles with wider patches. ${ }^{9}$ In passing, we note that recently it has been suggested that the entropy of mixing cubic and hexagonal layers makes stacking-disordered ice the stable phase for crystallites up to 
a size of at least $10^{5}$ molecules. ${ }^{18}$ Further simulation studies showed that the diamond nucleation free energy barrier reaches a minimum for particles with a patch width of about $30^{\circ}$. As patch width increases, the chemical potentials of the liquid and solid phases become closer (leading to a higher energy barrier), but the surface tension decreases (reducing the barrier) so that the minimum free energy barrier for diamond nucleation occurs for intermediate patches.

Here, we revisit the assembly of simple tetrahedral patchy particles from the fluid phase. We find that tetrahedral particles with narrow patches preferentially assemble into empty clathrate structures, whereas particles with intermediate patches form cubic and hexagonal diamond as shown in previous work. ${ }^{9}$ The formation of clathrates is attributed to kinetic factors whose origin is traced back to the thermodynamic stability of pentagonal rings as patch width decreases. These pentagonal rings can assemble into sII clathrate or into icosahedral clusters containing 100 particles grown from a central dodecahedral cage. Synthesis of colloidal particles with this simple geometry falls within today's experimentalist capabilities. ${ }^{19-21}$ Thus, it might be foreseeable that the assembly of clathrate structures using tetrahedral patchy particles might also be soon realizable in the lab.

\section{SIMULATION METHODS}

Interactions between tetrahedral particles are described by the Kern-Frenkel model ${ }^{22}$ which consists of a hard sphere particle of diameter $\sigma$ with four attractive sites or patches on the surface, each of which can form a bond with energy $-\epsilon$. Using $\sigma$ and $\epsilon$ as the length and energy units, this model has only two parameters: the patch half opening angle $\theta_{\max }$ and the interaction range $\Delta$, which can be independently tuned. The assembly behavior was investigated by means of Monte Carlo simulations in the NVT ensemble. First, we performed numerous exploratory simulations in a system with $N=1000$ particles, sweeping the parameter space formed by the patch width $\left(\cos \theta_{\max }=0.98-0.92\right)$ and the interaction range $(\Delta=0.03-0.24)$. Once the region where clathrates spontaneously form was identified, simulations on a much larger system with $N=10000$ were also performed to get further insight into the crystallization of clathrates.

We have chosen to perform NVT MC simulations with single particle moves at density $\rho \sigma^{3}=0.03$ and temperature $k_{B} T / \epsilon=0.116$, as is observed that in this condition a single crystal cluster is formed in reasonable computational times. Study of crystallization from a dilute phase by addition of single particles may lead to a faster crystallization and an easier identification of the polymorph formed than in simulations at higher densities close to that of diamond (conditions at which our preliminary simulations also predicted the formation of clathrates). Note that single particle MC moves produce an artificial dynamics, which only in specific cases (small displacement/rotation steps) can be mapped into Brownian dynamics. ${ }^{53}$ Previous work has also shown that the tendency of a low density liquid to gelation (which will slow or even prevent crystallization) might be artificially suppressed if collective motion is neglected. ${ }^{23}$ Luckily, at the selected $T$, bonds break and reform several times during the simulation. Collective motion can be mimicked using cluster moves designed to use in conjunction with MC or Brownian dynamics schemes but only approximately and at a considerable computational cost. ${ }^{24-26}$ The most rigorous route to simulate the dynamics in out-of-equilibrium processes involves performing Brownian dynamics simulations in which hydrodynamic interactions within the clusters are taken into account, something that is beyond the scope of this work.

All throughout this article, $\sigma$ and $\epsilon$ are used as the distance and energy units, respectively. Thus, temperature is expressed as $T^{*}=k_{B} T / \epsilon$, density is expressed as $\rho^{*}=\rho \sigma^{3}$, and pressure is expressed as $p^{*}=p \sigma^{3} / \epsilon$.

For the 4-patch KF model, the gas-liquid critical point $T$ is quite accurately predicted by $B_{2}^{*}=-4.92$, where $B_{2}^{*}=1-4(1$ $\left.-\cos ^{2} \theta_{\max }\right)\left((1+\Delta)^{3}-1\right)(\exp \beta \epsilon-1)$ is the reduced second virial coefficient.

The evolution of crystal growth was monitored using $\mathrm{CHILL}^{+}{ }^{28}$ This order parameter has the advantage over other ones that it is capable of distinguishing between hexagonal and cubic ice, and clathrate structures, identifying also interfacial particles. Given that some trajectories assemble into clathrate structures, we implemented also the order parameter proposed by Jacobson et al. ${ }^{29}$ to discriminate between the two most common clathrate structures, sI and sII. This order parameter is based on the identification of cages and vertex present in the different clathrate structures. ${ }^{29}$ Adopting the usual convention, each cage or polyhedron will be assigned a label $n_{i}^{m_{i}}$, where $i$ runs over the different types of polygonal faces forming the cage, $n_{i}$ is the number of edges of each type of polygonal face, and $m_{i}$ is the number of faces with $n_{i}$ edges. The cages that appear in sI and sII are the dodecahedron $\left(5^{12}\right)$, a tetradecahedron $\left(5^{12} 6^{2}\right)$, and a hexadecahedron $\left(5^{12} 6^{4}\right)$. Then, each vertex in the lattice is assigned four indices accounting for the number cages of each type converging at that site.

\section{RESULTS}

\section{A. Assembly "experiments"}

The summary of the crystallization behavior as a function of the interaction parameters is shown in Fig. 1. Consistent with previous work, our simulations indicate that the patch width has a much larger impact on the assembly behavior than the interaction range. Crystallization is achieved for patch widths narrower than about $40^{\circ}$,

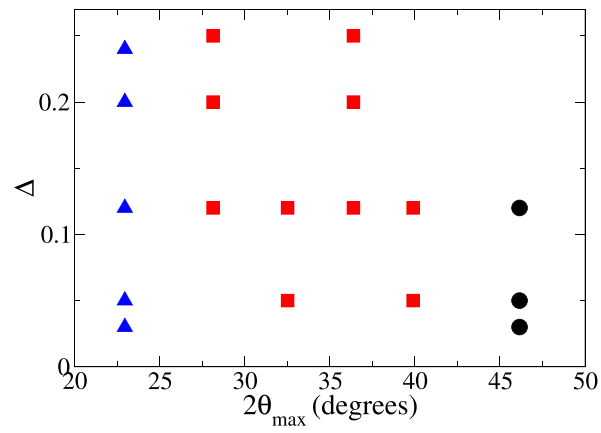

FIG. 1. Structure formed from the fluid phase as a function of model parameters, namely, interaction range $\Delta$ and patch width $2 \theta_{\max }$. Black circles represent the glass state, red squares represent cubic/hexagonal diamond, and blue triangles indicate that both diamond and clathrates are assembled. 
which is somewhat larger than the limit value reported previously. This is just a consequence of exploring more thermodynamic conditions and performing longer simulations. But, more interestingly, we find that the crystal formed is not always a mixture of cubic and hexagonal diamond as expected. For the narrowest patch, the assembly of diamond competes with the spontaneous formation of empty clathrates. The interaction range again seems to play a secondary role in the probability of the system crystallizing in either of these crystal structures.

With the aim of getting further insight into the crystallization of clathrate structures, we performed 10 independent simulations in a larger system with $\mathrm{N}=10000$ particles for the model with $\cos \theta_{\max }$ $=0.98$ and $\Delta=0.24$ at a density $\rho=0.03$. These simulations were performed at $T=0.126$, a temperature at which nucleation often starts from a single crystalline nucleus and occurs at reasonable simulation times. Our simulations indicate that different trajectories follow different crystallization paths. Three representative configurations of the aggregate formed at the end of the simulation are depicted in Figs. 2(d), 2(h), and 2(1). The particles in different crystal environments as identified with $\mathrm{CHILL}^{+}$are shown in different colors.

In the most common situation, the fluid crystallizes into an aggregate formed mainly of particles in clathratelike environments [Fig. 2(d)]. In these trajectories, crystallization coincides with a sudden rise in the number of clathrate particles [Fig. 2(a)]. There are also some particles at interfacial clathrate environments, which are obviously those located at the aggregate surface. On the contrary, the number of diamond particles is negligible throughout all the trajectories. Therefore, it can be concluded that nucleation starts from a clathrate nucleus that grows steadily.

In some other instances, both clathrate and cubic/hexagonal diamond coexist in the aggregate [Fig. 2(h)]. The evolution of the number of particles in different crystal environments shows that in the first nucleation stage the nucleus is formed almost exclusively of clathrate particles, and only after some time, the number of diamond particles also increases [Fig. 2(e)]. Consistent with previous

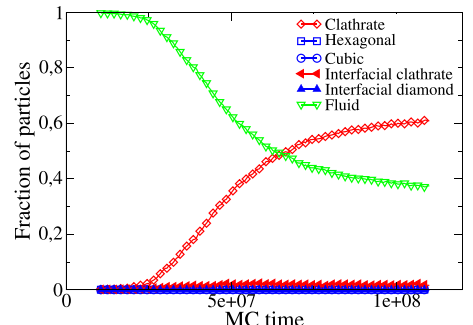

(a)

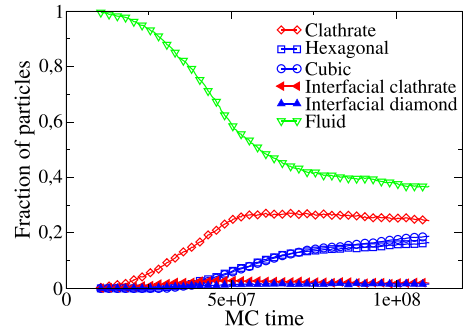

(e)

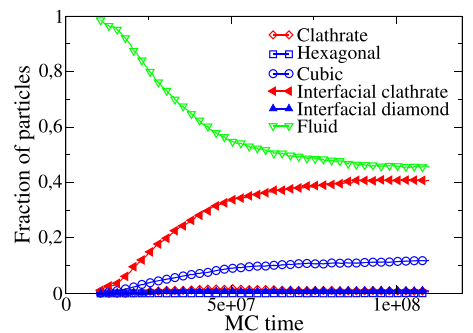

(i)

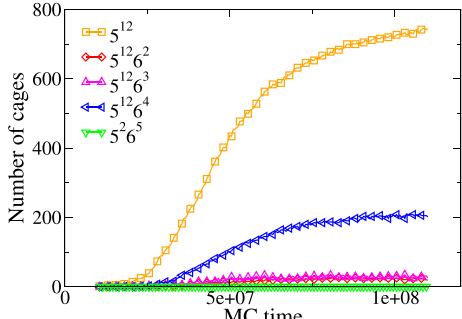

(b)

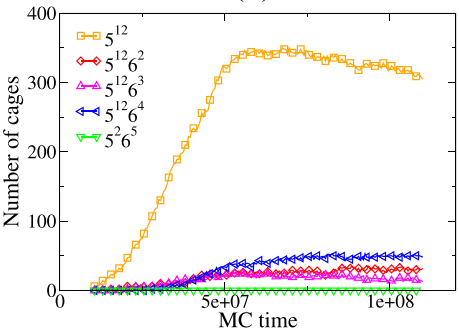

(f)

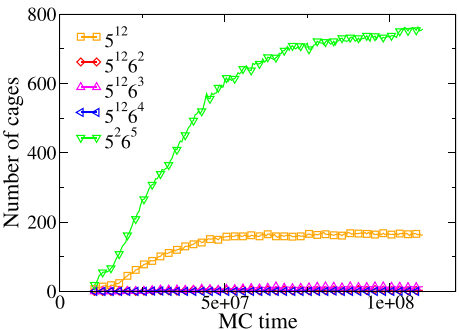

(j)

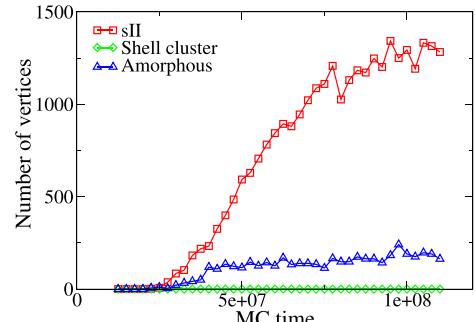

(c)

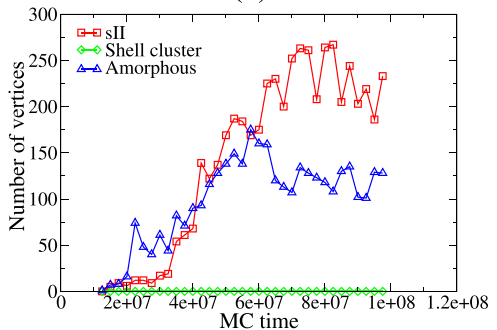

(g)

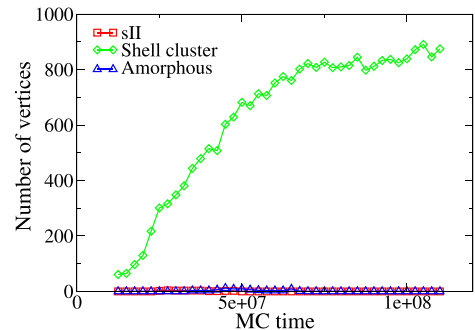

(k)

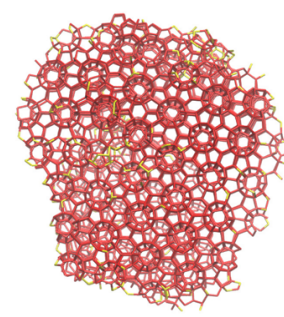

(d)

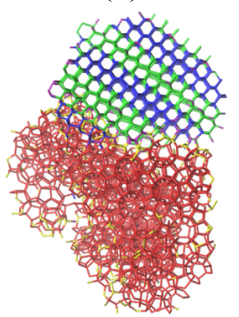

(h)

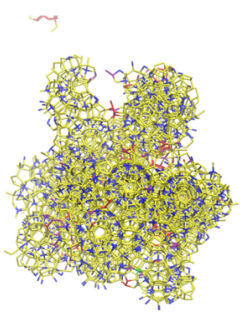

(1)

FIG. 2. Evolution of the number of particles in different crystal environments as identified using $\mathrm{CHILL}^{+}(\mathrm{a}),(\mathrm{e})$, and (i) and of the number of cages (b), (f), and (j) and vertices (c), $(\mathrm{g})$, and $(\mathrm{k})$ detected by Jacobson order parameter for clathrates, ${ }^{29}$ along three representative trajectories of the crystallization of a fluid of tetrahedral particles with $\cos \theta_{\max }=0.98$ and $\Delta=0.03$ at $T^{*}=0.116$. The final configuration along each of the three selected trajectories is also shown: (d) sll clathrate, (h) coexisting sll and diamond, and (I) an amorphous aggregate. Particles in different colors represent different crystal environments as identified with $\mathrm{CHILL}^{+}$: clathrate particles are shown in red, interfacial clathrate is shown in yellow, cubic diamond is shown in blue, hexagonal diamond is shown in green, and interfacial diamond is shown in purple. 
findings, ${ }^{9}$ this crystal consists of a random stack of cubic and hexagonal diamond. This is just as a consequence of cubic and hexagonal diamond presenting a similar nucleation free energy barrier, as well as almost identical free energy. Curiously, the number of clathrate particles remains almost constant shortly after that diamond growth starts. The fact that clathrate and diamond structures can coexist during relatively long simulation times suggests that their free energy might be similar, but the higher tendency of diamond to grow hints that diamond might be indeed the thermodynamically stable phase.

Finally, some trajectories assemble into a large aggregate whose structure seems disordered at first sight [Fig. 2(1)]. However, most of the particles in this structure are classified as cubic diamond or interfacial clathrate by $\mathrm{CHILL}^{+}$, both environments being evenly distributed over the aggregate and growing simultaneously (with the number of interfacial clathrate particles typically being more than three times higher than those in cubic diamond environments). Interestingly, a careful inspection reveals that particles are often arranged into an icosahedral three-shell cluster containing 100 particles and formed by two inner dodecahedral shells covered by a rhombicosidodecahedron [see Fig. 3(c)]. This structure can also be seen as formed by a central dodecahedral cage, surrounded by 20 barrelian-shaped pentagonal cages in which two pentagons are joined by five other particles forming five boat 6membered rings [see Fig. 3(d)]. By analogy with the notation often used to name the cages in clathrate structures and defined before, these cages are designated here $5^{2} 6^{5}$, although we should emphasize that the five six-edge faces are not planar (nor even nearly planar). As can be seen in Fig. 3(e), particles belonging to the central dodecahedral cage form three eclipsed and one staggered bond with its nearest neighbors and, thus, are identified as interfacial clathrate by $\mathrm{CHILL}^{+}$. Those belonging to the second dodecahedral shell form four staggered bonds, i.e., they have the same local environment as cubic diamond. Particles on the surface of the 100 cluster form two eclipsed and one staggered bond. They will be identified as interfacial clathrate or fluid depending on the fourth neighbor.

Further information about the structure of these ordered and disordered clathrates can be obtained using Jacobson order parameter that identifies cages and vertices within these lattices. ${ }^{29}$ This parameter is slightly modified here to include the $5^{2} 6^{5}$ cages that appear in the icosahedral three-shell cluster. Thus, vertices are now defined using five instead of four indices $v_{j k l m n}$, where the indices $j, k, l, m$, and $n$ are the number of $5^{12}, 5^{12} 6^{2}, 5^{12} 6^{3}, 5^{12} 6^{4}$, and $5^{2} 6^{5}$ cages converging at that vertex. Using this convention, sI clathrate contains vertices of types $v_{04000}$ and $v_{13000}$, sII of types $v_{04000}$ and $v_{13000}$, and the icosahedral shell cluster of types $v_{10003}$ and $v_{00004}$. Analysis of clathrate structures with this order parameter reveals that the clathrate assembled from the melt at these conditions is invariably of type sII, as evidenced by the large number of $5^{12} 6^{4}$ cages [Figs. 2(b) and 2(f)] and correct sII vertices [Figs. 2(c) and $2(\mathrm{~g})]$.

Regarding the amorphous aggregate, it contains a large number of dodecahedral $5^{12}$ and pentagonal $5^{2} 6^{5}$ cages [Fig. 2(j)] and only a tiny amount of $5^{12} 6^{2}, 5^{12} 6^{3}$, and $5^{12} 6^{4}$ cages, typical of sI, sII, and amorphous water clathrates. ${ }^{29,30}$ Consistently, the growth of the aggregate is not correlated with an increase of sI, sII, or amorphous clathrate vertices. On the contrary, there is a rather pronounced enhancement of the number of vertices compatible with the three shell icosahedral cluster [Fig. 2(1)]. By merging dodecahedral and pentagonal cages, we were able to identify more than 40 complete icosahedral shell clusters in this aggregate [see Figs. 3(a) and 3(b)]. These clusters are often joined by interpenetration, sharing a pentagonal cage. Two typical unions of icosahedral cluster are shown in Figs. 3(f) and $3(\mathrm{~g})$. Even though there are some regions where the clusters show some tendency to aggregate in an ordered way, there is really no order beyond the shell cluster. As can be seen in Fig. 3(b) the structure can be described as an amorphous aggregate of shell icosahedral clusters. We speculate that the lack of ordering of the cluster aggregates might be related to a possible increase in strain when merging shell clusters.

Interestingly, the stability of a three shell cluster with the same structure as the one spontaneously assembled here has been theoretically studied as a possible arrangement of the water molecules in the liquid state. ${ }^{31}$ In the case of water, this unit made of 100 particles is the central core of a larger icosahedral cluster with $\mathrm{N}=240$ molecules. Reference 32 suggested that the cluster can grow in three dimensions by adding more shells in the 12 faces, but it is more strained as cluster size increases. According to the author, such a growth on cooling qualitatively explains the origin of water density and pressure anomalies.

\section{B. Stability of clathrate structures}

The spontaneous formation of clathrates from tetrahedral particles with narrow patches raises the obvious question of whether they might become thermodynamically stable in this region of the parameter space. Even though the phase diagram of tetrahedral patchy particles has already been investigated, ${ }^{6-8}$ clathrates were not considered in these studies. Given that the distribution of nearest neighbors around a central particle exhibits deviations from the perfect tetrahedral arrangement of the particles, it is reasonable to think that clathrates are likely to be entropically disfavored over cubic and hexagonal diamond, in which patches point directly to the nearest neighbors (see Table I). Additionally, the density of clathrates is lower than that of hexagonal and cubic diamond, which makes them also enthalpically disfavored. In any case, we checked the stability of sI and sII clathrates by performing free energy calculations using the Einstein molecule approach. ${ }^{33-35}$ Initial clathrate structures were taken from Ref. 36, scaling the Cartesian coordinates to the appropriate interaction range. The phase diagrams for four sets of model parameters are shown in Fig. 4. As can be seen, clathrates are metastable for tetrahedral patchy particles over all the parameter space. They only become more stable than diamond at negative pressures, sII being slightly more stable than sI. The relative stability of clathrates with respect to diamond increases for small patches and large interaction ranges. Note, however, that diamond stability decreases for long ranges. Coincidentally, simulations of the phase diagram of water using TIP4P/2005 also found that sII is more stable than sI, becoming only more stable than the remaining solid phases at negative pressures. ${ }^{37}$ In the case of water, though, another clathrate structure $\mathrm{sH}$ was more stable than sI. However, $\mathrm{sH}$ is not even mechanically stable for patchy particles, as it involves local environments with large deviations from the tetrahedral arrangement. This is consistent with Ostwald's empirical observation that the crystal that nucleates first does not 


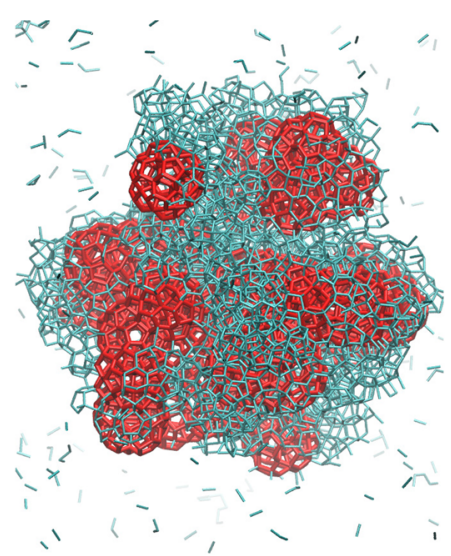

(a)
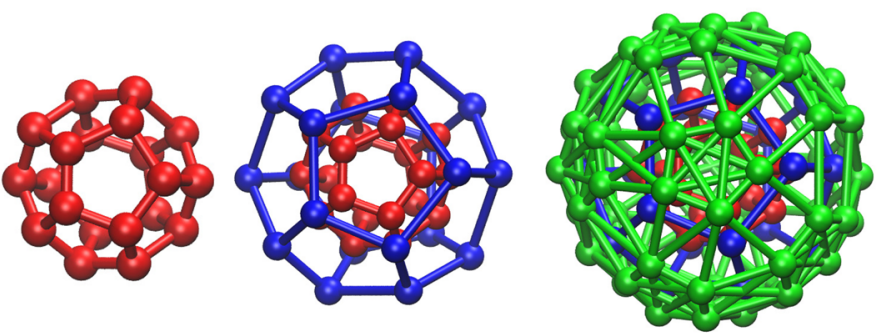

(c)
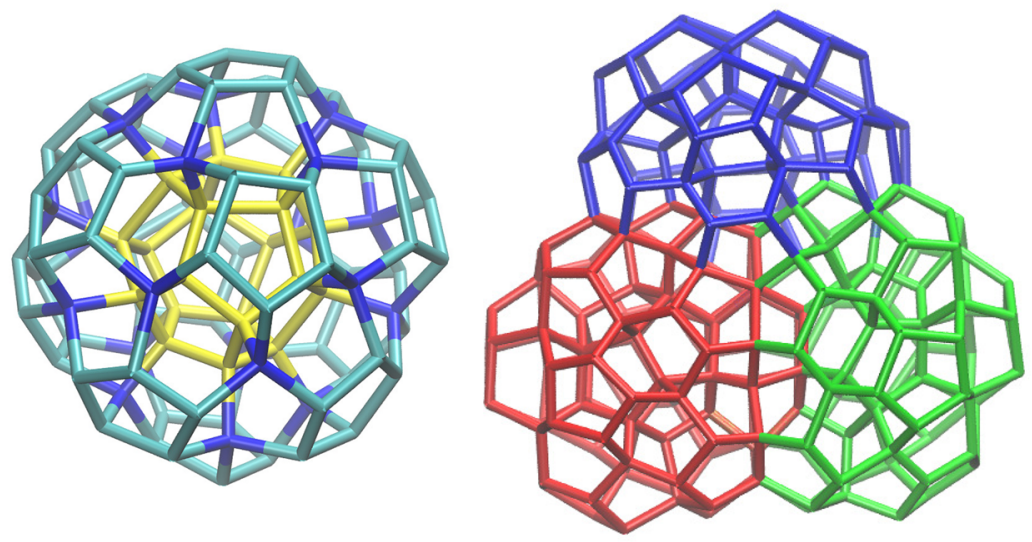

(f)

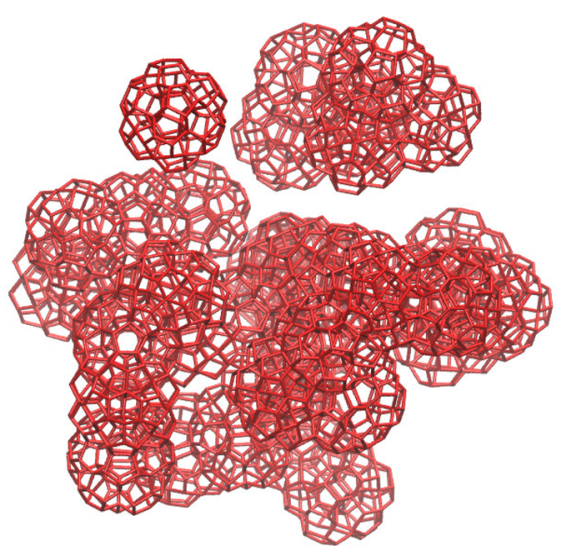

(b)

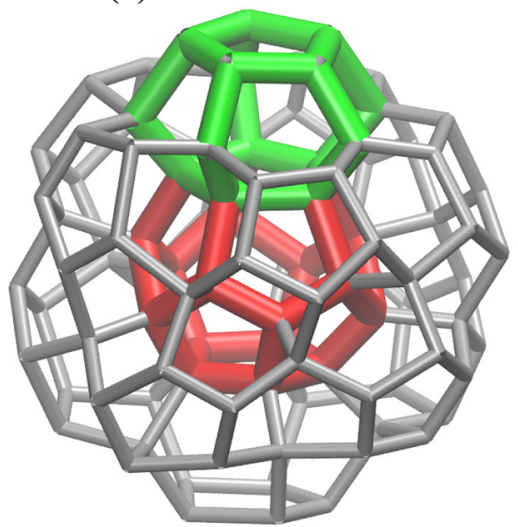

(d)

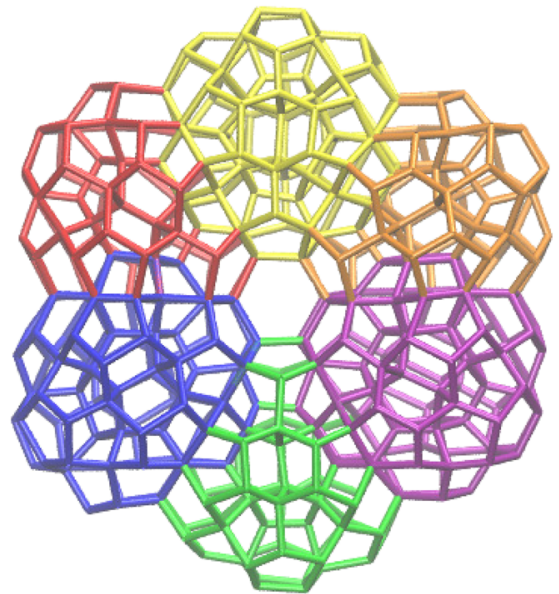

(g)

FIG. 3. [(a) and (b)] Two different representations of the amorphous aggregate. In (a), particles belonging to shell clusters are shown in red and the remaining particles are shown in cyan. In (b), particles that do not belong to complete shell clusters are removed to aid visualization. (c) Structure of the three shells forming a 100-particle cluster that is often found in the amorphous clathrate aggregate. (d) The two types of cages formed in the cluster are a central dodecahedral cage shown in red, surrounded by twelve $5^{2} 6^{5}$ barrelianlike cages (one of which is highlighted in green). (e) $\mathrm{CHILL}^{+}$identifies particles belonging to the central dodecahedral cage (yellow) as interfacial clathrate (they form three eclipsed and one staggered bond), those in the second dodecahedral shell (dark blue) as cubic diamond (four staggered bonds) and those belonging to the external rhombicosidodecahedron (cyan) as fluid (particles in these sites form two eclipsed and one staggered bond) or as interfacial clathrate depending on the fourth bond. Two typical forms of joining the shell clusters: (f) three interpenetrated shell clusters and $(\mathrm{g})$ six interpenetrated shell clusters around a $5^{2} 6^{5}$ cage. 
TABLE I. Wyckoff positions in sl and sll structures, as well as the angles formed by the neighbors in these sites.

\begin{tabular}{lcc}
\hline \hline Crystal & Wyckoff site & Angles with neighbors \\
\hline sI & $24 \mathrm{k}$ & $124.628,106.70,106.70,106.12,106.12,105.10$ \\
& $16 \mathrm{i}$ & $110.565,110.565,110.565,108.353,108.353,108.353$ \\
& $6 \mathrm{c}$ & $110.74,110.743,108.839,108.839,108.839,108.839$ \\
\hline \multirow{2}{*}{ sII } & $96 \mathrm{~g}$ & $119.86,108.63,108.63,107.96,105.59,105.59$ \\
& $32 \mathrm{e}$ & $111.55,111.55,111.55,107.30,107.30,107.30$ \\
& $8 \mathrm{a}$ & $109.47,109.47,109.47,109.47,109.47,109.47$ \\
\hline \hline
\end{tabular}

necessary coincide with the thermodynamically stable phase, a finding that was rationalized by Stranski and Totomanow by stating that the phase that forms first is that separated from the fluid by a lower free energy barrier. ${ }^{38}$ Thus, we can conclude that patchy particles with small patches crystallize into sII, not because it is the thermodynamically stable phase but because the free energy barrier separating this crystal from the fluid is possibly lower than that of diamond.

\section{Why are clathrate structures kinetically favored?}

Once we have seen that clathrates are always metastable, the next question is then why they are preferentially formed over the stable diamond phase for particles with small patches. For this purpose, we analyzed the properties of the fluid phase. We performed simulations in the NVT ensemble at $\rho^{*}=0.03$ for particles with angular patch widths within $\cos \theta_{\max }=0.90-0.99$ and constant

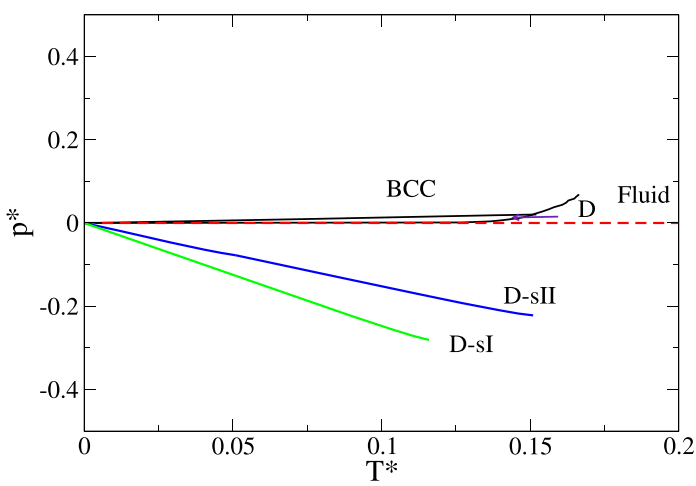

(a)

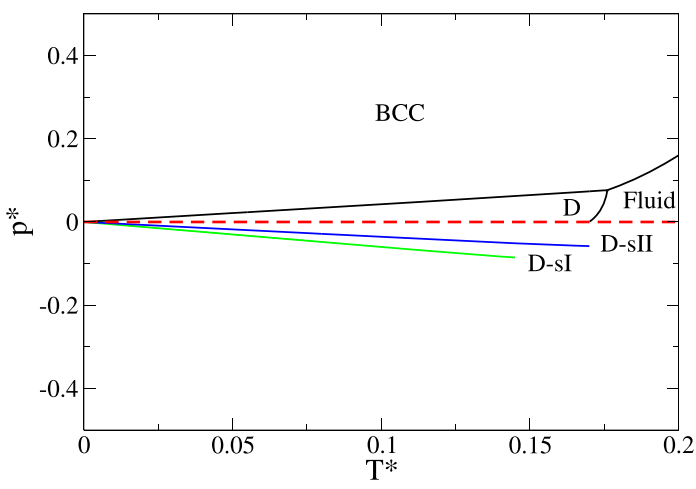

(c)

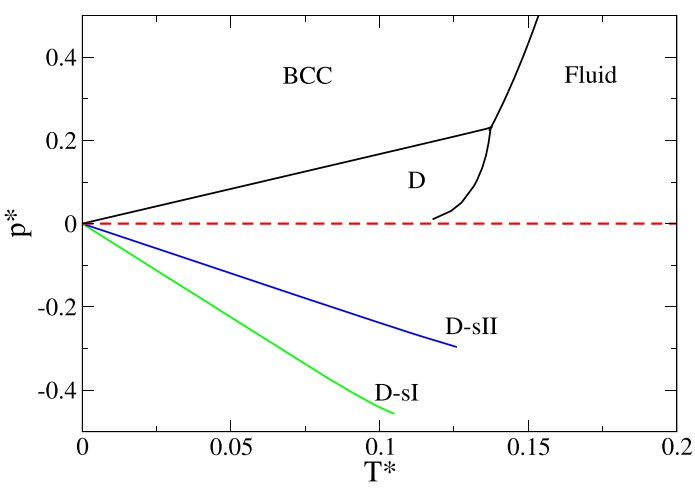

(b)

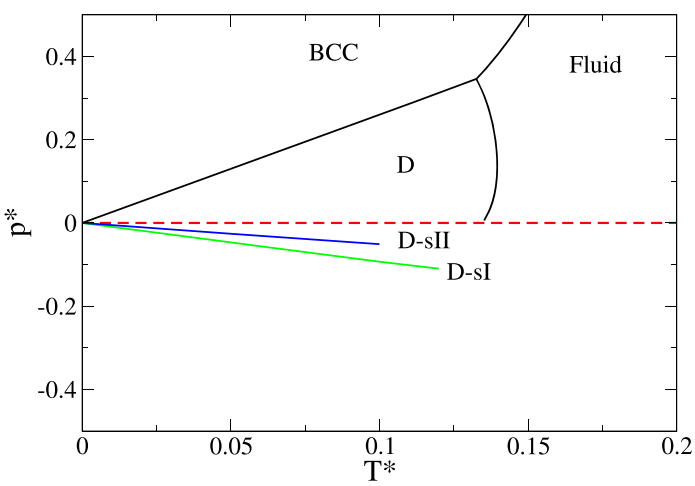

(d)

FIG. 4. Phase diagram of tetrahedral patchy particles for different angular patch widths and interaction ranges. Clathrates sl and sll are always metastable with respect to the fluid phase. They become more stable than diamond at negative pressures, sll clathrate being more favorable than sl. (a) $\cos \theta_{\max }=0.98, \Delta=0.24$. (b) $\cos \theta_{\max }=0.98$, $\Delta=0.03$. (c) $\cos \theta_{\max }=0.92, \Delta=0.24$. (d) $\cos \theta_{\max }=0.92, \Delta=0.03$. 
interaction range $\Delta=0.03$. The bonding probability exhibits a discontinuity at a temperature that decreases with patch width indicating the onset of nucleation [see Fig. 5(a)]. The number of bonded rings was analyzed as a function of patch width and at a constant value of bonding probability $p_{b}=0.16$ (slightly smaller than the $p_{b}$ value at which nucleation starts). Comparing systems in thermodynamic equilibrium with the same number of bonds (same $p_{b}$ ) but different $\theta_{\max }$ values allows us to highlight unambiguously the connection between ring formation and patch width. As can be seen in Fig. 5(b), four-membered rings disappear for particles with small patches (with $\cos \theta_{\max }>0.97$ ) due to geometric constraints. On the contrary, both 5- and 6-membered rings are more likely for particles with small patches, but the growth of 5-member rings is more significant than that of 6-membered rings. The dilute fluid prepares itself to self-assemble clathrates when the patch size decreases.

The available volume for bonding in planar $n$-membered rings can be written, to first approximation, as

$$
V_{b}^{n-r i n g}=2 \pi \sigma^{2}\left(\cos \theta_{0}-\cos \theta\right) \Delta
$$

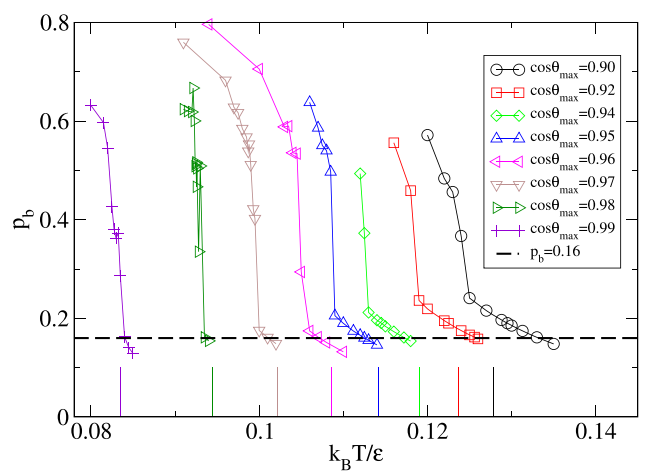

(a)

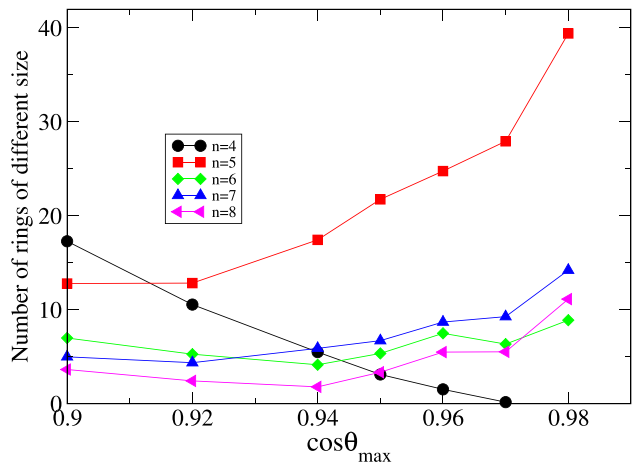

(b)

FIG. 5. (a) $T$ dependence of the bond probability for different $\cos \theta_{\max }$ values at $\rho=0.03$. A clear discontinuity is observed in all curves in concomitance with gas-liquid phase separation and/or nucleation. Data for $T$ smaller than the discontinuity are provided as a reference, being the system slowly aging. The lines at the bottom indicate the model critical $T$ estimated by the proposed relation $B_{2}^{*}\left(T_{c}\right)=-4.92{ }^{27}$ (b) Number of rings (defined as closed paths along bonds, starting and ending in the same particle) for different values of $\cos \theta_{\max }$, all at $p_{b}=0.16$ [see the dashed line in panel (a)]. where $\theta_{0}$ corresponds to the smallest patch width compatible with ring formation. Considering the simple case of planar rings, this angle can be estimated as $(109.47-\alpha) / 2$, where $109.47^{\circ}$ is the tetrahedral angle and $\alpha$ is the interior angle of the regular polygon with $n=4,5,6, \ldots$, edges. According to this, the minimum angles for 4-, 5-, and 6-membered rings are $\cos \theta_{0}^{4}=0.9856, \cos \theta_{0}^{5}$ $=0.9999$, and $\cos \theta_{0}^{6}=0.996$. This means that 5 - and 6-membered (both boat and chair) rings can even be formed for very directional bonds. Thus, this very simple argument explains why 4-membered rings are not formed for particles with narrow patches, but it is by itself not enough to explain why the number of 5-membered rings increases more rapidly than 6 -membered rings as patches become narrower.

In general, the configurational part of the partition function of a cluster of particles of size $n$ can be written as

$$
\begin{aligned}
Q_{n}= & \frac{1}{n ! \sigma^{3 n}} \int^{\prime} d \vec{r}_{1}, \ldots, d \vec{r}_{n} d \Omega_{1}, \ldots, d \Omega_{n} \\
& \times \exp \left[-\beta V\left(\vec{r}_{1}, \vec{r}_{2}, \ldots, \vec{r}_{n}, \Omega_{1}, \ldots, \Omega_{n}\right)\right],
\end{aligned}
$$

where $\vec{r}_{1}, \ldots, \vec{r}_{n}$ are the Cartesian coordinates of the particles and $\Omega_{1}, \ldots, \Omega_{n}$ are their orientations and where we have arbitrarily set the thermal de Broglie length to the unit distance $\sigma$. The prime sign in the integration limit indicates that only points in phase space for which the cluster does not break into disconnected smaller clusters are considered. For well defined morphologies, one can calculate the integral for a specific set of ordered patches and particles and multiply the result by a combinatorial factor, $\Omega_{n}^{c o m b}$, accounting for all the possible arrangements of the particles with that morphology. For chains, the partition function can be conveniently rewritten by using relative positions with respect to the first particle in the chain,

$$
Q_{n}^{\text {chain }}=\frac{\Omega_{n}^{\text {chain }}}{n ! \sigma^{3 n}} e^{-\beta \epsilon(n-1)} V \int^{\prime \prime} d \overrightarrow{r_{21}}, \ldots, d \overrightarrow{r_{n 2}} d \Omega_{1}, \ldots, d \Omega_{n},
$$

where $V$ is the volume of the system. The double prime in the integral indicates that integration is limited to phase space points for which the cluster-now with a specific set of ordered patches and particle labels-retains its integrity. The combinatorial factor $\Omega_{n}^{\text {chain }}$ for particles with $f$ patches can be inferred by recurrence. Two particles with $f$ patches can bind to each other in $f^{2}$ different ways. For each one of these arrangements, the chain can bind an additional particle in $(2 f-2) \times f$ different ways $[(2 f-2)$ is the number of available patches in the two free ends and $f$ is that in the new attached particle]. Particles forming a chain of length $(n-1)$ can be arranged in $(n-1) ! / 2$ different ways, where the factor 2 avoids overcounting of chains with the same sequence of particles from right to left and from left to right. Since the new particle can be located in $n / 2$ locations, we have

$$
\begin{aligned}
\Omega_{n}^{\text {chain }} & =\frac{\Omega_{n-1}^{\text {chain }}[f(2 f-2)] n}{2} \\
& =\frac{f^{2}}{2}[f(f-1)]^{n-2} n ! .
\end{aligned}
$$

For simplicity, we assume that successive bonds are decoupled so that the integral in Eq. (3) can be split into a product of $(n-1)$ integrals, one for each bond $V_{b}$. Supposing that translations and 
rotations can also be decoupled in the KF model, the configurational integral for each bond can be approximated by

$$
\begin{aligned}
V_{b} & =4 \pi\left[\frac{1-\cos \theta_{\max }}{2}\right]^{2} \frac{(\sigma+\Delta)^{3}-\sigma^{3}}{3} \\
& =\pi\left(1-\cos \theta_{\max }\right)^{2}\left(\sigma^{2} \Delta+\sigma \Delta^{2}+\frac{1}{3} \Delta^{3}\right) .
\end{aligned}
$$

We note, since we are going to use this value in the following, that for the case $\Delta=0.03$, the bonding volume is given by $V_{b}=0.0971\left(1-\cos \theta_{\max }\right)^{2}$. Putting everything together, the partition function of a chain of $n$ particles can be written as

$$
Q_{n}^{\text {chain }}=\frac{V}{\sigma^{3 n}} \frac{f^{2}}{2}[f(f-1)]^{n-2} V_{b}^{n-1} e^{-\beta \epsilon(n-1)} .
$$

Once the partition function is known, it is possible to evaluate the probability of observing that cluster in a simulation. Indeed, this probability is directly proportional to the value of its partition function, ${ }^{40,41}$

$$
N_{n}^{\text {chain }}=Q_{n}^{\text {chain }}\left(\frac{N_{1} \sigma^{3}}{V}\right)^{n},
$$

which only requires $Q_{n}^{\text {chain }}$ and the monomer number density $\rho_{1}=N_{1} / V$. For the case of chains considered here, this gives

$$
N_{n}^{\text {chain }}=\frac{V}{\sigma^{3 n}} \frac{f^{2}}{2}[f(f-1)]^{n-2} V_{b}^{n-1} e^{-\beta \epsilon(n-1)}\left(\frac{N_{1} \sigma^{3}}{V}\right)^{n} .
$$

The number of chains of size $n, N_{n}^{\text {chain }}$, and $\rho_{1}$ can be measured in equilibrium simulations of the fluid, and it allows one to double check the quality of the simulation data and, at the same time, the quality of the proposed theoretical partition function (i.e., the evaluation of the integral as product of independent bonding volumes) by comparing the left and right hand sides of the following equation for different $\cos \theta_{\max }$ values:

$$
\frac{N_{n}^{\text {chain }} e^{\beta \epsilon(n-1)}}{V \rho_{1}^{n} V_{b}^{n-1}}=\frac{f^{2}}{2}[f(f-1)]^{n-2} .
$$

Note that the $T$ at which the simulation is performed is irrelevant since it enters in a known way and it is factored out. As shown in Fig. 6, the agreement between the simulation and the approximation of independent bonds worsens for longer chain lengths. However, for all $n$, there is clearly no patch width dependence in $Q_{n}^{\text {chain }}$ beside the $\left(1-\cos \theta_{\max }\right)^{2}$ included in $V_{b}$, as predicted by the theory.

Let us move now to the case of a ring of size $n$. Its partition function can be written as

$$
Q_{n}^{\text {ring }}=\frac{\Omega_{n}^{\text {ring }}}{\sigma^{3 n} n !} V \Omega^{\prime} e^{-\beta \epsilon n},
$$

where now the number of bonds is $n$ and the integral in the phase space, $\Omega^{\prime}$, is over all configurations in which all particles have two bonds.

The number of possible ways of forming a ring with $n$ distinguishable particles with $f$ distinguishable patches is given by

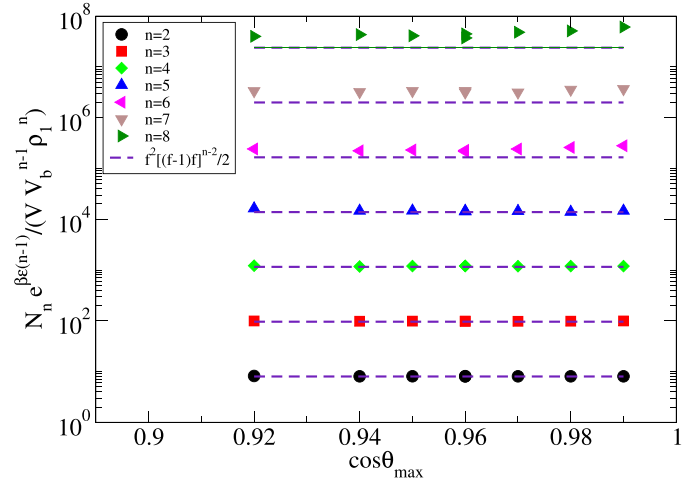

FIG. 6. Left and right hand sides of Eq. (9). The left side (symbols) is evaluated from simulation data at $\rho=0.03\left[\Delta=0.03\right.$ and hence $V_{b}=0.0971(1-$ $\left.\cos \theta_{\max }\right)^{2}$ ]. Specifically, we calculate the number of chain forming clusters $N_{n}^{\text {chain }}$ of different sizes $n$ and the monomer (chain of length 1) number density from equilibrium simulation. The right hand side (dashed lines) is the expected $f^{2}[f(1-f)]^{n-2} / 2$ theoretical prediction. Note that no dependence on the patch width $\theta_{\max }$ is observed (beside the one included in $V_{b}$ ). Since the topology of the cluster is fixed, the number of bonds is always $n-1$ and thus the Boltzmann factor entering in the partition function is a priori known, rendering the value of the simulation $T$ irrelevant.

$$
\begin{aligned}
\Omega_{n}^{\text {ring }} & =\frac{(n-1) !}{2}\left[f^{2} \times[(f-1) f]^{n-2} \times(f-1)^{2}\right] \\
& =\frac{(n-1) !}{2}\left[f^{n}(f-1)^{n}\right] .
\end{aligned}
$$

This expression is obtained considering that there are $f^{2}$ different ways of creating the first bond between two particles. For each one of these $f^{2}$ configurations, there are $f(f-1)$ possibilities of adding each of the $n-2$ following bonds (or equivalently each of the $n-2$ other particles). Finally, the last particle must bind with the first one. It has $f-1$ sites available for binding and must connect to one of the $f-1$ sites available for bonding in the first particle. Thus, patches contribute a term proportional to $f^{2}[f(f-1)]^{n-2}(f-1)^{2}=[f(f-1)]^{n}$. The term $(n-1) ! / 2$ accounts for the number of different rings of distinguishable particles. Finally, the partition function can be written as

$$
Q_{n}^{r i n g}=\frac{f^{n}(f-1)^{n}}{2 n \sigma^{3 n}} V \Omega^{\prime} e^{-\beta \epsilon n}
$$

where

$$
\Omega^{\prime}=\int^{\prime} d \overrightarrow{r_{21}}, \ldots, d \overrightarrow{r_{n 1}} d \Omega_{1}, \ldots, d \Omega_{n} .
$$

Now, the prime indicates that the integral is restricted to those configurations in which all the particles have two bonds. The evaluation of $\Omega^{\prime}$ is less straightforward in the case of rings. It can be approximated by $V_{b}^{n-1} R$, where $R$ counts the number configurations for which, when $n-1$ bonds are formed, a last bond that closes the loop is automatically present. Thus, the number of $n$-membered rings is given by

$$
N_{n}^{r i n g}=\frac{f^{n}(f-1)^{n}}{2 n \sigma^{3 n}} V \Omega^{\prime} e^{-\beta \epsilon n}\left(\frac{N_{1} \sigma^{3}}{V}\right)^{n},
$$




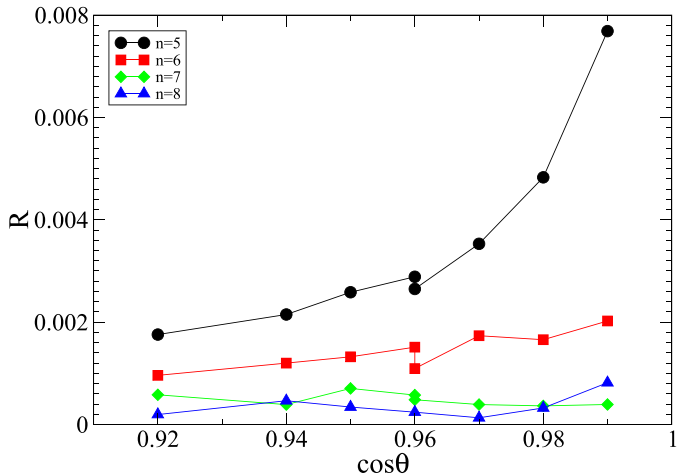

FIG. 7. Probability $R$ that the position and orientation of the first and last particles in a chain allow for the formation of a bond, transforming the chain into a ring, as a function of $\cos \theta_{\max }$ [evaluated as shown in Eq. (15)]. Here, $\Delta=0.03$ and hence $V_{b}=0.0971\left(1-\cos \theta_{\max }\right)^{2} ; \rho_{1}$ and $N_{n}^{\text {ring }}$ are calculated from MC simulations at $\rho=0.03$ and $T$ selected such that $p_{b}(T)=0.175$.

from where $R$ can be evaluated,

$$
R=\frac{N_{n}^{r i n g} e^{\beta \epsilon n} 2 n}{V \rho_{1}^{n} V_{b}^{n-1} f^{n}(f-1)^{n}} .
$$

As can be seen in Fig. 7, when $n=5$ and $n=6, R$ increases significantly with the angular patch width $\cos \theta_{\max }$, explaining the preferential formation of pentagonal (and to less extent hexagonal) rings in particles with small patches. These results indicate that the almost perfect match between the internal angle of the pentagon and the tetrahedral angle is the reason why pentamers are preferentially formed for tetrahedrally patchy particles with highly directional interactions.

\section{SUMMARY AND CONCLUSIONS}

In summary, we have found that the assembly of tetrahedral patchy particles can be governed by tuning the patch width. Whereas particles with large patches $\left(2 \theta_{\max } \gtrsim 40^{\circ}\right)$ lead to disordered glass states, particles with intermediate size patches $\left(20^{\circ} \lesssim 2 \theta_{\max } \lesssim 40^{\circ}\right)$ assemble into a diamond crystal, and those with small patches $\left(2 \theta_{\max } \lesssim 20^{\circ}\right)$ show a competition between diamond and clathrate structures.

A more extensive study of the nucleation of clathrates from a low density fluid of tetrahedral particles with narrow patches shows that there are several nucleation paths. Whereas in some trajectories the system assembles into large sII clathrate aggregates, in some other instances, nucleation starts from a sII nucleus, diamond nucleating later on the surface on this nucleus. Finally, the remaining trajectories follow a rather different path, in which the system assembles into a rather disordered aggregate formed by small icosahedral three-shell clusters. Taking a general perspective from the three different scenarios, it seems that nucleation often starts from dodecahedral cages. This is a consequence of the higher tendency of the fluid to aggregate into five-membered rings as patch width decreases. These cages can grow into sII clathrate or into an icosahedral shell cluster. Given that the second shell of particles in the icosahedral cluster is in almost perfect tetrahedral environment (they are identified as cubic diamond by $\mathrm{CHILL}^{+}$), this structure is probably less strained than that of small sII fragments. We speculate that this is the reason why the formation of shell cluster might be favored in some trajectories. Free energy calculations evidence that clathrate structures are never thermodynamically stable for tetrahedral particles regardless of their patch size or interaction range. Thus, nucleation of clathrates must be the result of kinetic factors, linked to the increasingly lower free energy of pentagonal rings-compared to hexagonal and larger rings-as the patch width decreases.

The results presented here also shed light on the propensity of water molecules to form in the liquid state a significant fraction of five membered rings. This is expected since a mapping of several water classical potentials into a KF model suggests an equivalent $\cos \theta_{\max } \gtrsim 0.92 .{ }^{13}$ Pentagonal rings have indeed received attention in the past both as candidates of low-density regions topologically distinct from the nucleating icelike (diamond) structures, providing resilience to crystallization, ${ }^{42}$ and for their role in promoting a decrease in the density on cooling ${ }^{31}$ (and of the other thermodynamic anomalies slaved to the existence of a temperature of maximum density ${ }^{43-45}$ ). In a very recent study based on the TIP4P- $2005^{46}$ potential, a structural model based on fused dodecahedral cages for low density water was investigated. ${ }^{47}$ Although molecular dynamics simulations predict that these clusters are rather unstable, the calculated spectral behavior and scattering behavior are consistent with the experimental data. Interestingly, another recent study of heterogeneous nucleation using the coarse-grained monoatomic $\mathrm{mW}$ water model reported that, under slow nucleation conditions, water formed nanostructures with a high proportion of pentagonal rings, similar to those found here. ${ }^{48}$

As a consequence of the fivefold symmetry axes (which are incompatible with crystalline growth), present both in the dodecahedral and barrelianlike pentagonal cages, it seems reasonable to think that it might be possible to use these subunits to build a quasicrystal. Indeed, dodecahedral and pentagonal cages were used in previous work to decorate a three-dimensional Penrose quasilattice with the aim of finding a tetracoordinated quasicrystal. ${ }^{49}$ In future work, it would be interesting to study whether the 100 particle clusters identified here can be arranged into extended periodic or aperiodic ordered structures.

The results presented here are of high relevance for those seeking to produce open colloidal crystals. Assembly of clathrates from patchy particles has been often assumed to be rather challenging due to the complexity of their unit cell. Consistent with this, all the soft matter systems that have been found to assemble into clathrates involve some degree of complexity, e.g., using mixtures of patchy colloid $^{50}$ or oscillating isotropic models ${ }^{51}$ in simulations and triangular bipyramid nanoparticles interacting via DNA strands in experiments. ${ }^{52}$ Here, we propose a much simpler route for producing these complex crystals.

\section{ACKNOWLEDGMENTS}

This work was funded by the Agencia Estatal de Investigación and the Fondo Europeo de Desarrollo Regional (FEDER) under 
Grant Nos. FIS2015-72946-EXP (AEI) and FIS2017-89361-C3-2-P (AEI/FEDER, UE) and by MIUR-PRIN 2018 under Grant No. 2017Z55KCW. E.G.N. acknowledges useful discussions with Jon Doye.

\section{REFERENCES}

${ }^{1}$ G.-R. Yi, D. J. Pine, and S. Sacanna, J. Phys.: Condens. Matter 25, 193101 (2013).

${ }^{2}$ E. Duguet, C. Hubert, C. Chomette, A. Perro, and S. Ravaine, C. R. Chim, 19, 173 (2016).

${ }^{3}$ M. A. Boles, M. Engel, and D. V. Talapin, Chem. Rev. 116, 11220 (2016).

${ }^{4}$ A. S. Keys, T. Chen, and S. C. Glotzer, Langmuir 21, 11547 (2005).

${ }^{5}$ J. P. K. Doye, A. A. Louis, I.-C. Lin, L. R. Allen, E. G. Noya, A. W. Wilber, H. C. Kok, and R. Lyus, Phys. Chem. Chem. Phys. 9, 2197 (2007).

${ }^{6}$ F. Romano, E. Sanz, and F. Sciortino, J. Phys. Chem. B 113, 15133 (2009).

${ }^{7}$ F. Romano, E. Sanz, and F. Sciortino, J. Chem. Phys. 132, 184501 (2010).

${ }^{8}$ E. G. Noya, C. Vega, J. P. K. Doye, and A. A. Louis, J. Chem. Phys. 132, 234511 (2010).

${ }^{9}$ F. Romano, E. Sanz, and F. Sciortino, J. Chem. Phys. 134, 174502 (2011).

${ }^{10}$ I. Saika-Voivod, F. Romano, and F. Sciortino, J. Chem. Phys. 135, 124506 (2011).

${ }^{11}$ G. Doppelbauer, E. G. Noya, E. Bianchi, and G. Kahl, Soft Matter 8, 7768 (2012).

${ }^{12}$ N. Dorsaz, L. Filion, F. Smallenburg, and D. Frenkel, Faraday Discuss. 159, 9 (2012).

${ }^{13}$ I. Saika-Voivod, F. Smallenburg, and F. Sciortino, J. Chem. Phys. 139, 234901 (2013).

${ }^{14}$ F. Smallenburg, L. Filion, and F. Sciortino, Nat. Phys. 10, 653 (2014).

${ }^{15}$ F. W. Starr and F. Sciortino, Soft Matter 10, 9413 (2014).

${ }^{16}$ N. Patra and A. V. Tkachenko, Phys. Rev. E 98, 032611 (2018).

${ }^{17}$ F. Sciortino, Eur. Phys. J. B 64, 505 (2008).

${ }^{18}$ L. Lupi, A. Hudait, B. Peters, M. Grünwald, R. G. Mullen, A. H. Nguyen, and V. Molinero, Nature 551, 218 (2017).

${ }^{19}$ Q. Chen, S. C. Bae, and S. Granick, Nature 469, 381 (2011).

${ }^{20}$ Y. Wang, Y. Wang, D. R. Breed, V. N. Manoharan, L. Feng, A. D. Hollingsworth, M. Weck, and D. J. Pine, Nature 491, 51 (2012).

${ }^{21}$ Z. Gong, T. Hueckel, G.-R. Yi, and S. Sacanna, Nature 550, 234 (2017).

${ }^{22}$ N. Kern and D. Frenkel, J. Chem. Phys. 118, 9882 (2003).

${ }^{23}$ S. Whitelam, E. H. Feng, M. F. Hagan, and P. L. Geissler, Soft Matter 5, 1251 (2009).

${ }^{24}$ S. Whitelam and P. L. Geissler, J. Chem. Phys. 127, 154101 (2007).

${ }^{25}$ S. Babu, J.-C. Gimel, T. Nicolai, and C. D. Michele, J. Chem. Phys. 128, 204504 (2008).
${ }^{26}$ A. Prabhu, S. Babu, J. S. Dolado, and J.-C. Gimel, J. Chem. Phys. 141, 024904 (2014).

${ }^{27}$ G. Foffi and F. Sciortino, J. Phys. Chem. B 111, 9702 (2007).

${ }^{28}$ A. H. Nguyen and V. Molinero, J. Phys. Chem. B 119, 9369 (2015).

${ }^{29}$ L. C. Jacobson, M. Matsumoto, and V. Molinero, J. Chem. Phys. 135, 074501 (2011).

${ }^{30}$ L. E. Bove and U. Ranieri, Philos. Trans. R. Soc., A 377, 20180262 (2019).

${ }^{31}$ O. Loboda and V. Goncharuk, Chem. Phys. Lett. 484, 144 (2010).

${ }^{32}$ R. Ludwig, Angew. Chem., Int. Ed. 40, 1808 (2001).

${ }^{33}$ C. Vega and E. G. Noya, J. Chem. Phys. 127, 154113 (2007).

${ }^{34}$ E. G. Noya, M. M. Conde, and C. Vega, J. Chem. Phys. 128, 154507 (2008).

${ }^{35}$ C. Vega, E. Sanz, J. L. Abascal, and E. G. Noya, J. Phys.: Condens. Matter 20, 153101 (2008).

${ }^{36}$ See https://homepage.univie.ac.at/michael.leitner/lattice/index.html for the atomic structure of clathrates sI, sII, and $\mathrm{sH}$.

${ }^{37}$ M. M. Conde, C. Vega, G. A. Tribello, and B. Slater, J. Chem. Phys. 131, 034510 (2009).

${ }^{38}$ I. N. Stranski and D. Totomanow, Z. Phys. Chem. 163A, 399 (1933).

${ }^{39}$ X. Mao, Q. Chen, and S. Granick, Nat. Mater. 12, 217 (2013).

${ }^{40}$ T. L. Hill, An Introduction to Statistical Thermodynamics (Courier Corporation, 1986).

${ }^{41}$ F. Sciortino, in Proceedings of the International School of Physics Enrico Fermi, Course 193, Soft Matter Self-Assembly, edited by C. Likos, F. Sciortino, E. Zaccarelli, and P. Ziherl (IOS Press, Amsterdam and SIF, Bologna, 2016).

${ }^{42}$ J. Russo and H. Tanaka, Nat. Commun. 5, 3556 (2014).

${ }^{43}$ S. Sastry, P. G. Debenedetti, F. Sciortino, and H. E. Stanley, Phys. Rev. E 53, 6144 (1996).

${ }^{44}$ P. H. Poole, I. Saika-Voivod, and F. Sciortino, J. Phys.: Condens. Matter 17, L431 (2005).

${ }^{45}$ V. Holten, C. Qiu, E. Guillerm, M. Wilke, J. Ricka, M. Frenz, and F. Caupin, J. Phys. Chem. Lett. 8, 5519 (2017).

${ }^{46}$ J. L. Abascal and C. Vega, J. Chem. Phys. 123, 234505 (2005).

${ }^{47}$ G. Camisasca, D. Schlesinger, I. Zhovtobriukh, G. Pitsevich, and L. G. M. Pettersson, J. Chem. Phys. 151, 034508 (2019).

${ }^{48}$ Y. Dai and X. Xu, J. Phys. Chem. C 122, 15729 (2018).

${ }^{49}$ J. Peters and H.-R. Trebin, Phys. Rev. B 43, 1820 (1991).

${ }^{50}$ D. F. Tracey, E. G. Noya, and J. P. Doye, "Programming patchy particles to form complex ordered structures" (unpublished).

${ }^{51}$ M. Engel, P. F. Damasceno, C. L. Phillips, and S. C. Glotzer, Nat. Mater. 14, 109 (2015).

${ }^{\mathbf{5 2}}$ H. Lin, S. Lee, L. Sun, M. Spellings, M. Engel, S. Glotzer, and C. A. Mirkin, Science 355, 931 (2017).

${ }^{53}$ F. Romano, C. De Michele, D. Marenduzzo, and E. Sanz, J. Chem. Phys. 135(12), 124106 (2011). 